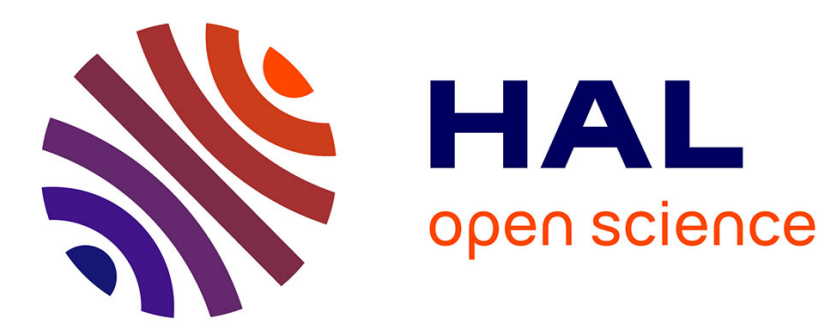

\title{
Analytical Survey of Highlight Detection in Color and Spectral Images
}

\author{
Haris Ahmad Khan, Jean-Baptiste Thomas, Jon Yngve Hardeberg
}

\section{To cite this version:}

Haris Ahmad Khan, Jean-Baptiste Thomas, Jon Yngve Hardeberg. Analytical Survey of Highlight Detection in Color and Spectral Images. International Workshop on Computational Color Imaging, Mar 2017, Milan, Italy. 10.1007/978-3-319-56010-6_17 . hal-01672528

\section{HAL Id: hal-01672528 https://hal.science/hal-01672528}

Submitted on 5 Jan 2018

HAL is a multi-disciplinary open access archive for the deposit and dissemination of scientific research documents, whether they are published or not. The documents may come from teaching and research institutions in France or abroad, or from public or private research centers.
L'archive ouverte pluridisciplinaire HAL, est destinée au dépôt et à la diffusion de documents scientifiques de niveau recherche, publiés ou non, émanant des établissements d'enseignement et de recherche français ou étrangers, des laboratoires publics ou privés. 


\title{
Analytical survey of highlight detection in color and spectral images
}

\author{
Haris Ahmad Khan ${ }^{1,2}$, Jean Baptiste Thomas ${ }^{1,2}$, and Jon Yngve Hardeberg ${ }^{1}$ \\ ${ }^{1}$ The Norwegian Colour and Visual Computing Laboratory, \\ NTNU - Norwegian University of Science and Technology, Gjøvik, Norway \\ ${ }^{2}$ Le2i, FRE CNRS 2005, Univ. Bourgogne Franche-Comté, Dijon, France \\ \{haris.a.khan, jean.b.thomas, jon.hardeberg\}@ntnu.no
}

\begin{abstract}
Detection of highlights is a prominent issue in computer vision, graphics and image processing. Applications which require object properties measurement or rendering are affected by specular reflection since the models assume matte diffusing surfaces most of the time. Hence, detection, and sometimes removal, of specular reflection (highlights) in an image may be critical. Several methods are proposed for addressing this issue. In this paper, we present a review and analysis of these techniques in color and spectral images.
\end{abstract}

Keywords: image analysis, highlights detection, specular reflection, diffuse reflection, spectral imaging.

\section{Introduction}

The process of extracting information from an image, and its transformation into a useful representation, enables the description of intrinsic characteristics of objects in the scene. Barrow et al. 1 introduced the term "intrinsic images" and suggested that the function of the human visual system at its earlier stage is to determine the orientation of the illumination and the surface being observed. The authors describe such details of scenes in term of shading and reflectance images, which are collectively denoted as intrinsic images. There are other intrinsic properties as well, including shading, reflectance, diffuse reflection components and specular reflection components. Diffuse reflection is caused by scattering of light in all directions after hitting the surface, while specular reflection occurs when incident light is reflected in a single direction. Lee et al. 2 presented a neutral interface reflection model by examining the light reflection problem through the use of bidirectional spectral-reflectance distribution function (BSRDF) for specifying both incident and reflected beam geometry. They proposed that specular reflection is identical to scene illuminant in color while diffuse reflection contains the intrinsic properties of the surface. There can be significant variations in appearance of a surface in presence of specular reflection, and they appear as an additional surface property which is not intrinsic [3] 4 .

We are interested in the detection of specular reflection in images. Several computer vision applications such as 3D reconstruction, object detection, recognition, target tracking, and dichromatic editing use the intrinsic information and 
are mostly based on the assumption of the surfaces having perfect diffusion. In most of such algorithms, specular reflections and highlights are termed as outliers 5. However, the presence of specular reflection is unavoidable in most of the real world scenes since the materials and surfaces not only possess diffuse reflection but also show specular reflections, which is explained in the Dichromatic Illumination Model (DIM) [6. Hence, the assumption of absence of specular reflection for such algorithms introduce constraints and reduce their robustness. There is a loss of details in case of specular reflection, for example texture and color of surface being observed. Highlight removal is often considered as an inpainting problem [7-10]. This methodology is implemented after identification of highlights. In this paper, we discuss the methods for highlight detection. Discussion about removal of specular highlights is out of scope of this paper.

As an example, Figure 1 shows an image of a printed circuit board. The information about connectivity of the circuit is totally washed out in the area which is under direct specular reflection. In addition, there are highlight spots on the metallic surfaces of diodes and resistors. Such spots follow the DIM and create ambiguity concerning the intrinsic characteristics of the object surface. It may thus be desirable to remove the effect of highlights from the image for recovery of information.

Highlight detection algorithms can be classified into various categories on the basis of data being used for input. For color images, we propose two major categories, namely the single-image and multi-images based techniques, presented in Section 2 and 3, respectively. Furthermore, a relatively recent development in imaging technology is spectral imaging. The problem of specular highlights occurs also in spectral images, therefore we have included it in this review as another category (Section 4). However, this review focuses only on close range

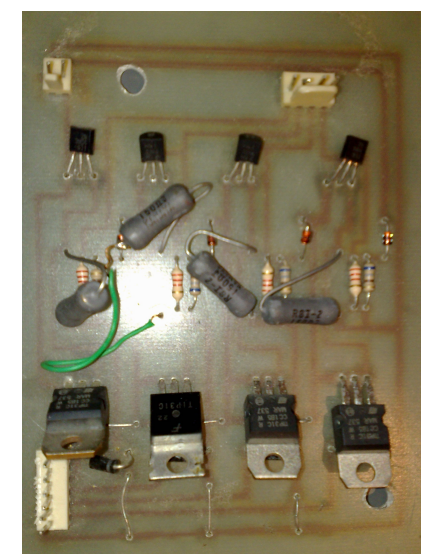

Fig. 1. Image of printed circuit board with transistors, resistors and diodes. Specular highlights can be observed in the highly saturated areas, where it washes out the information. In addition, specular spots are also observed on the metallic parts and resistors. 
spectral imaging and does not address remote sensing images. In Section 5, we discuss and compare key features of the presented algorithms, before concluding.

\section{Single color image based techniques}

We have defined two major categories of algorithms to detect and remove specularities in a single color image. Those categories use either the color space analysis, or spatial information analysis.

The techniques based on color space analysis treat an image pixel by pixel. Klinker et al. [11] classified color pixels in the categories of diffuse, highlight and saturated pixels. A diffuse pixel is defined as a pixel containing only the body reflectance (although the color of body is influenced by scene illuminant), a highlight pixel contain both body and specular reflections while a saturated pixel is created when a highlight pixel exceeds the maximum measurable light intensity of camera sensor. Klinker et al. [11] analyzed the color histogram and observed that the specular and diffuse components from a uniform surface form a skewed $\mathrm{T}$ shape. To separate these components, convex polygon fitting technique is used in their work. Linking color space with DIM [6] is also proposed [12] 13]. This color information is used to separate reflection components by fitting it into a dichromatic plane.

Transformation into other color spaces for detection of specularities is also a technique where the characteristics of an adequate color space are exploited. Schlüns and Teschner [14] 15] transformed the image from RGB to Y'U'V' color space. Bajcsy et al. [16] proposed S-space for analysis of variation in color of objects. There are three orthogonal basis functions in S space named $S_{0}, S_{1}$ and $S_{2}$. The $S_{0}$ basis function corresponds to specular reflection in S space. A datadriven color space called SUV color space was introduced by Mallick et al. [17. Yang et al. 18] proposed Ch-CV space. This color space is spanned by maximum chromaticity (Ch) and the coefficient of variation (CV) in RGB. Yang et al. [19] proposed separation of specular and diffuse components in HSI color space as further improvement in their already proposed Ch-CV space. Recently, Akashia and Okatani 20] proposed an optimization technique for sparse non-negative matrix factorization for the identification of specular reflections in an image.

In spatial information based techniques, detection of specularities is performed through the use of local information in an image. Tan et al. 21] introduced Maximum Chromaticity-Intensity Space to differentiate between the maximum intensity and maximum chromaticity in an image. A pseudo-diffuse component image is created, which is later utilized for separation of specular reflection from the image. The ratio of intensities and colors amongst neighbouring pixels is preserved in the pseudo-diffuse image and is called the specular free image. The specular free image is obtained by Yoon et al. 22] through subtracting the minimum value for each channel from the input image. Shen and Cai 23 introduced a modified specular free image by adding an offset to the subtraction method provided in 22]. This offset can either be constant for the whole image 24] or can be varying for each pixel [23. In [4], intensity logarith- 
mic differentiation is used on both the specular-free and the input image for determination of diffuse pixels. Using this method, performance of the technique of creation of specularity free image is improved for highly textured surfaces. However, the position of highlights should be known for applying this method. Liu et al. 25] proposed the preservation of surface color saturation by initially producing a specular free image and then increasing the achromatic component of diffuse chromaticity. It is important to note that in all the specularity free image creation techniques, the input image is normalized for illuminant. Hence, the illuminant should either be known or should be estimated first before applying those techniques.

Yang et al. 26] 27. treat the specular pixels as noise and use a bilateral filter for smoothing the maximum fraction of color components. In this way, the noise caused by specular pixels is eliminated. Kim et al. 28] observed that a diffuse pixel has low intensity in, at least, one channel. They called it the "dark channel" and proposed that the dark channel of an image contains no specular reflection. Their technique uses a maximum a-posteriori formulation that helps in the recovery of specular reflection and chromaticity. An et al. 29] proposed the pure diffuse pixel distribution model. This model is built on the assumption that there is at-least one purely diffuse pixel for each material in the scene. Shen and Zheng [30] assumed that some area of a surface contain only the diffuse reflection. They define the range value for an image as maximum minus minimum intensity for a surface and observed that the intensity ratio between maximum value of a pixel and range value is independent of the geometry of the surface. With the assumption for certain pixels to be diffuse, specular components from remaining pixels are computed through their proposed model.

Highlight detection techniques based on a single color image are practical as they do not require any additional hardware and data. However a problem associated with such algorithms is that they rely on image statistics and are based on strong prior assumptions. Therefore, such methods are not robust for change in imaging environment but works reasonably well when the required conditions are fulfilled.

\section{Multiple images based techniques}

The use of multiple images for separation of reflectance components from the scene is proposed in a number of studies. Since highlights are not intrinsic properties of an image, they can occur at any point and are dependant on the viewing angle. The direction of illumination also has its impact on the location of highlight spots. A surface area of an image which is affected by highlight in one image can appear as a diffuse surface if the viewing angle, or the illumination direction is changed. Based on this phenomenon, multiple images based highlight removal techniques are proposed in the literature, which require a number of images, captured using different imaging conditions.

Lee and Bajcsy [31] proposed the use of Lambertian consistency, which states that the Lambertian reflection does not vary in brightness and spectral contents 
with change in the viewing angle while the specular reflection changes the behaviour. They defined the spectral distance as euclidean distance between two colour points in a three-dimensional space. Minimum spectral distance is calculated to detect the inconsistency in color among two images of same scene but with different viewing angle. Sato and Ikeuchi [32] introduced temporal-color space analysis by using a moving light source. Lin and Shum [33] used different illuminations for the same scene, and then proposed linear basis functions for separating diffuse and specular components. Lin et al. 34] used stereo images for the detection of specularities. Weiss [35] acquired an image sequence with varying specularities and used maximum likelihood estimation by assuming that the change in illumination gives rise to sparse filter outputs.

Feris et al. [36] used flash images taken with same point of view but different positions of flash for recovering the diffuse component. Agrawal et al. 37. proposed a method for image enhancement by using two images of a scene. One is taken with flash and one without flash. Reflection from the flash image is removed by using a gradient projection scheme.

Chen et al. 38 reconstructed the specular field by using histograms of the same image but with different intensities. Yang et al. 39 proposed statistical methods for removal of specularities from stereo images. They assume nonoverlapping highlight regions in their method. Wang et al. [40] used three cameras for taking images of transparent plastic package containing tablets. They normalized the acquired images and then generated an image consisting of average intensities of corresponding pixels. In this way, pills are retained with higher intensity while varying regions of specular reflection are removed. Generation of specularity map from video sequence is proposed by Prinet et al. 41]. Recently, Wang et al. [42] proposed the use of light field imaging technology for capturing multiple views of a scene and then used that information for detection of specularities in the scene.

Nayar et al. 43 noticed that the specular reflection is highly polarized and proposed the use of polarization filter to separate the diffuse and specular reflections. They used a polarization filter by placing it in front of a camera and observed that by rotating the polarization filter, the brightness of diffuse materials is not changed, while the specular reflection is changed, since it is highly polarized, and varies following a cosine function. Polarization based methods are also proposed by Wolff [44, 45, Kim et al. 46, Atkinson and Hancock [47, 48, Müller [49, Umeyama and Godin [50, Lamond et al. [51] and Zhang et al. 52].

Although the above mentioned specularity detection techniques are able to show good performance, the major limitation associated with them is the availability of multiple images of the same scene with varying illumination direction or viewing angle. This limitation causes those techniques to become less practical compared to the approaches which are based on a single image. 


\section{Spectral image based techniques}

Hyperspectral and multispectral imaging has been used extensively for remote sensing. Recently, with advancement in sensor technology, spectral imaging is widely available for imaging of objects at shorter distance. Such imaging comes with the problem of highlights, the same as in the case of color images. However, since spectral images contain more data compared to a color image, therefore highlight detection techniques are not exactly the same.

Bochko and Parkkinen [53] proposed probabilistic principal component analysis for detection of spectral and diffuse parts in images. Fu et al. 554 proposed orthogonal subspace projection (OSP) and dichromatic model for specularityfree representation of hyperspectral images. OSP results in projecting the radiance and illumination spectrum being orthogonal to each other in the subspace. In this way, the illuminant spectra is removed from mixed spectra and a spectral image without highlights is obtained. It is important to note that prior information about illuminant is required in this method. Koirala et al. [55] proposed spectral unmixing of end-members for separation of specular and diffuse components in spectral images. Spectral end-members consist of pure spectra corresponding to objects in a scene. Spectral unmixing method is widely used in hyperspectral remote sensing where the end-members correspond of pure spectra of land cover classes. In the method by Koirala et al. 555, Automated target generation program (ATGP) is employed for selection of end-members. When the scene illuminant is known, then the initial target detected by ATGP is the illuminant spectra. In case of unknown illuminant, a pixel with maximum value along the whole spectra is considered as the initial target. Using constrained energy minimization, the diffuse part of the image is identified.

\section{Analysis of various specularity detection methods}

In Table 1, we compare the characteristics of various specularity detection techniques. In these tables, different techniques are divided in general categories. The concept behind the technique is briefly defined along with the general assumptions being made. Use of DIM is common in the specularity detection techniques but there are some algorithms that do not use this model, so it is also given in the table. White balancing (WB) and image segmentation (Seg.) is also a constraint in such algorithms. We make analysis of various categories of highlight detection algorithms on the basis of the above expressed factors. Finally, general remarks about strength of those techniques is provided. 


\begin{tabular}{|c|c|c|c|c|c|c|c|}
\hline 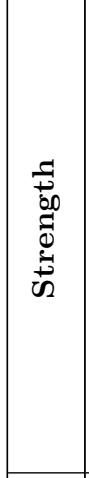 & 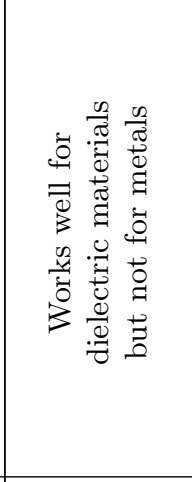 & 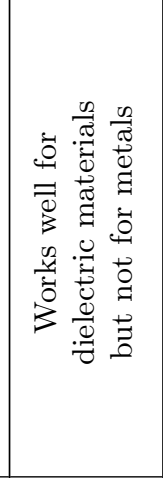 & 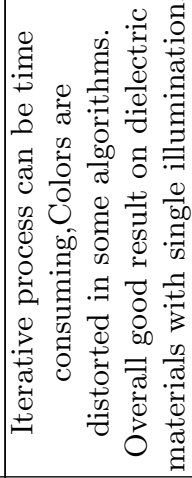 & 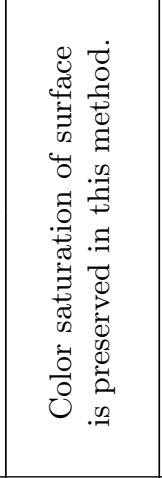 & 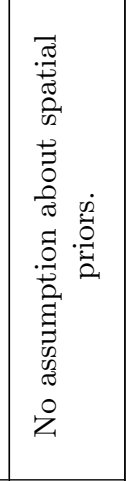 & 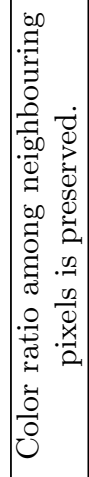 & 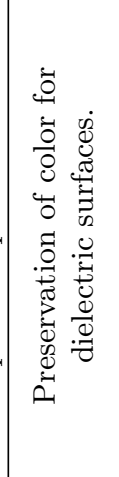 \\
\hline 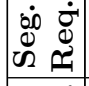 & 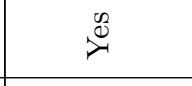 & $\underbrace{\infty}$ & $\sum_{\nu}^{\infty}$ & $\stackrel{\circ}{z}$ & $\stackrel{\circ}{z}$ & $\underset{\nu}{\mathscr{D}}$ & $\underbrace{\infty}$ \\
\hline $\mid \begin{array}{ll}n & \dot{8} \\
3 & \stackrel{d}{*}\end{array}$ & $\underbrace{\infty}$ & $\underbrace{\infty}$ & $\sum_{\lambda}^{\mathscr{D}}$ & $\underbrace{\mathscr{D}}_{-\infty}$ & $\stackrel{\mathscr{D}}{\nearrow}$ & $\underset{\nu}{\mathscr{C}}$ & 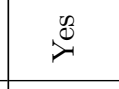 \\
\hline 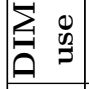 & $\underset{\nu}{y}$ & $\underset{\nu}{\mathscr{D}}$ & $\underset{\nu}{\mathscr{E}}$ & $\underset{\nu}{\mathscr{\nu}}$ & $\underset{\nu}{\mathscr{D}}$ & $\overbrace{}^{\infty}$ & $\stackrel{\circ}{z}$ \\
\hline 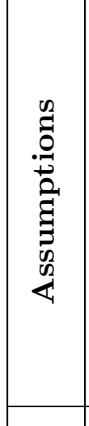 & 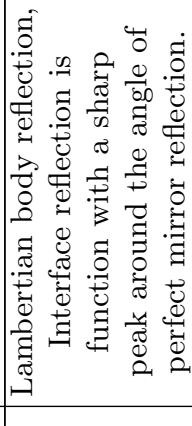 & 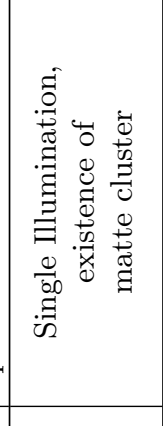 & 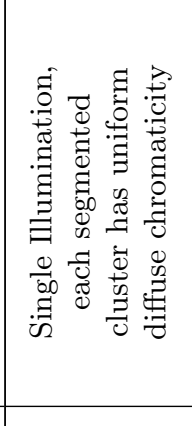 & 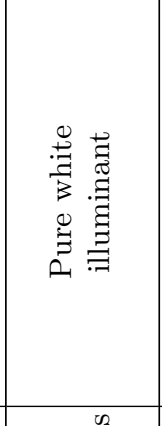 & 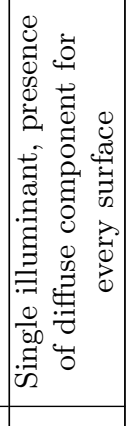 & 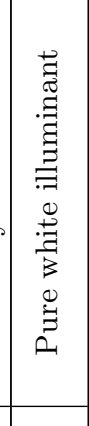 & 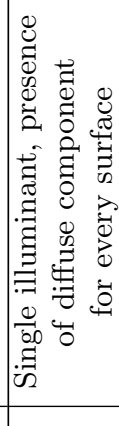 \\
\hline $\begin{array}{l}\overrightarrow{0} \\
\stackrel{0}{0} \\
\ddot{0} \\
ن\end{array}$ & 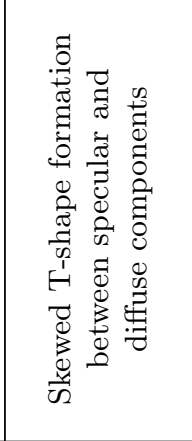 & 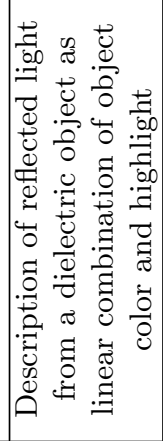 & 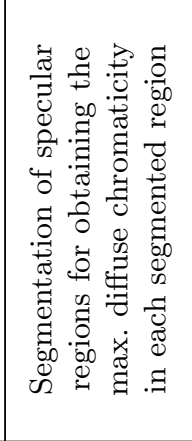 & 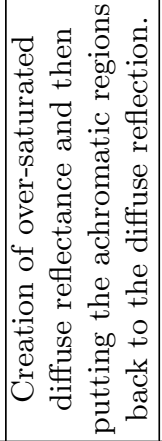 & 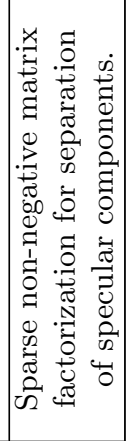 & 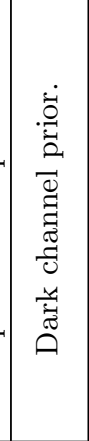 & 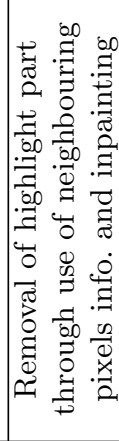 \\
\hline 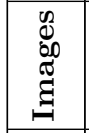 & $\begin{array}{l}\frac{0}{60} \\
: \overrightarrow{0}\end{array}$ & $\begin{array}{l}\frac{0}{60} \\
\stackrel{9}{9}\end{array}$ & $\begin{array}{l}\frac{0}{60} \\
:=\end{array}$ & $\begin{array}{l}\frac{0}{60} \\
\stackrel{9}{6}\end{array}$ & $\begin{array}{l}\frac{0}{\infty 0} \\
\ddot{*}\end{array}$ & $\begin{array}{l}0 \\
\stackrel{0}{60} \\
\stackrel{g}{\Rightarrow}\end{array}$ & $\begin{array}{l}\frac{0}{60} \\
\stackrel{7}{7}\end{array}$ \\
\hline 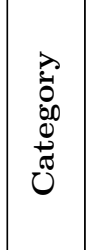 & 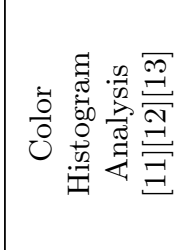 & 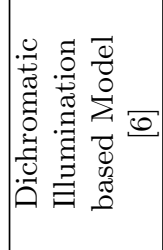 & 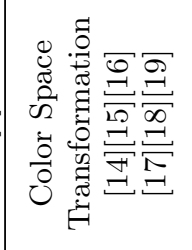 & 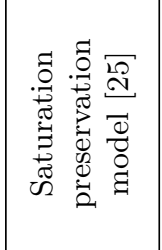 & 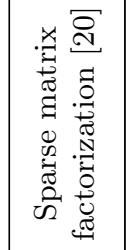 & 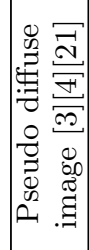 & 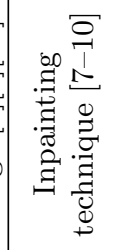 \\
\hline
\end{tabular}




\begin{tabular}{|c|c|c|c|c|c|}
\hline 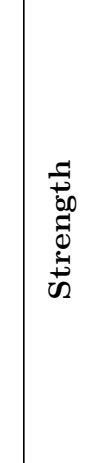 & 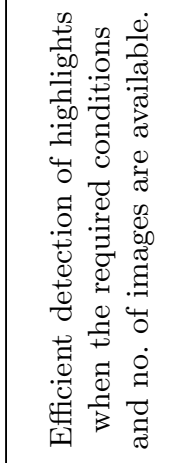 & 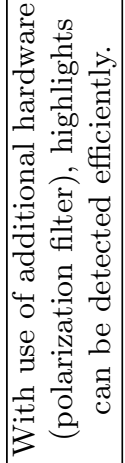 & 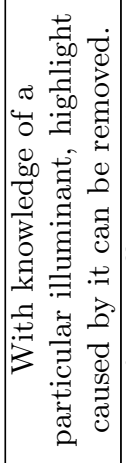 & 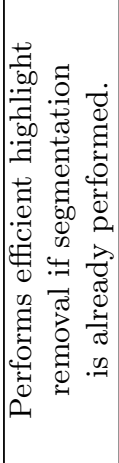 & 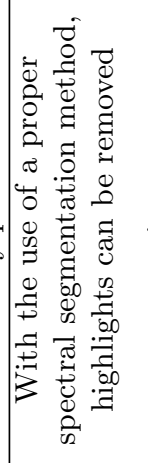 \\
\hline 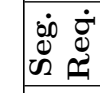 & 1 & 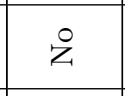 & $\stackrel{\circ}{z}$ & on & $\stackrel{\circ}{z}$ \\
\hline $\begin{array}{l}m \\
\dot{8} \\
3\end{array}$ & 1 & $\stackrel{\circ}{z}$ & $\underbrace{\infty}_{\nu}$ & $\stackrel{\circ}{z}$ & $\stackrel{\circ}{z}$ \\
\hline 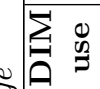 & $\underbrace{\infty}$ & $\overbrace{\lambda}^{\infty}$ & $\sum^{\infty}$ & $\sum^{\infty}$ & $\underbrace{\infty}$ \\
\hline 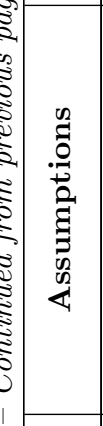 & 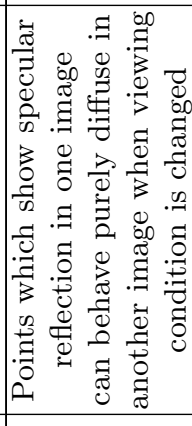 & 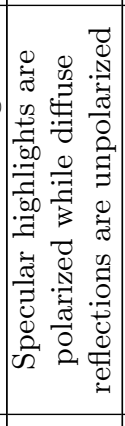 & 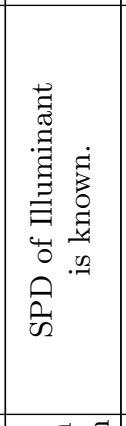 & 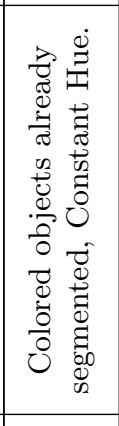 & 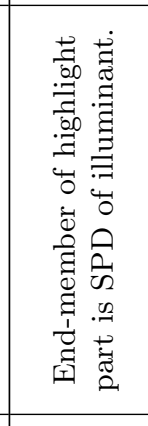 \\
\hline $\begin{array}{l}\overrightarrow{0} \\
\stackrel{0}{0} \\
0 \\
0 \\
0\end{array}$ & 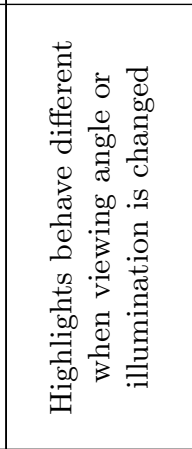 & 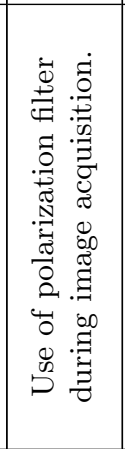 & 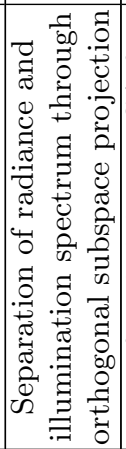 & 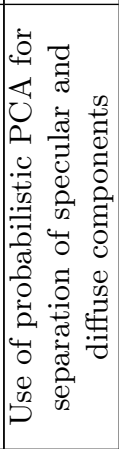 & 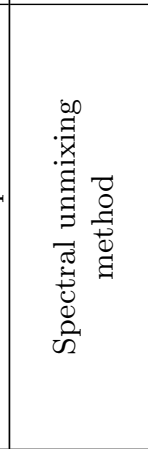 \\
\hline 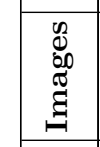 & 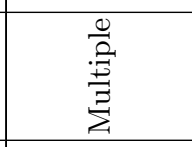 & 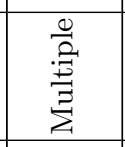 & 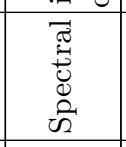 & 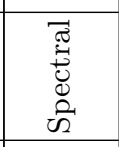 & 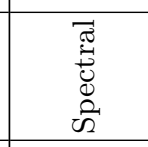 \\
\hline 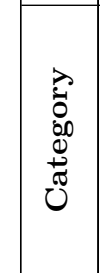 & 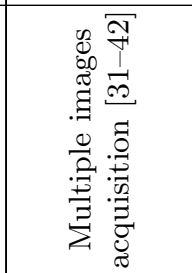 & 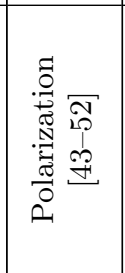 & 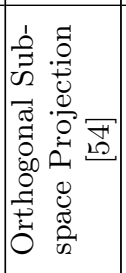 & 芯凪 & 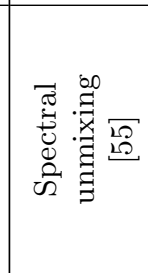 \\
\hline
\end{tabular}




\section{Conclusion}

In this paper, we provide a survey of highlight detection algorithms in color and spectral images. In most of the literature, results from detection and removal of highlights is presented qualitatively while comparing with other methods. Although qualitative analysis provide a general overview, quantitative measurement should also be used to provide a fair comparison.

Generally, strong assumptions and priors are used for highlight detection. However, the highlight detection is still able to provide much of the useful information and is therefore a critical issue in image analysis. We also review state-of-the-art on highlight detection in spectral images. There is less work done on it and much is yet to be explored. The amount of spectral information being acquired in spectral imaging can certainly provide benefit for highlight detection and is still an open area of research.

\section{References}

[1] H. Barrow and J. Tanenbaum, "Recovering intrinsic scene characteristic from images," Computer Vision System, pp. 3-26, 1978.

[2] H. C. Lee, E. J. Breneman, and C. P. Schulte, "Modeling light reflection for computer color vision," IEEE Transactions on Pattern Analysis and Machine Intelligence, vol. 12, pp. 402-409, Apr 1990.

[3] P. Tan, L. Quan, and S. Lin, "Separation of highlight reflections on textured surfaces," in proceedings of the 2006 IEEE Computer Society Conference on Computer Vision and Pattern Recognition, vol. 2 of CVPR '06, (Washington, DC, USA), pp. 1855-1860, IEEE Computer Society, 2006.

[4] R. T. Tan and K. Ikeuchi, "Separating reflection components of textured surfaces using a single image," in Digitally Archiving Cultural Objects, (Boston, MA), pp. 353-384, Springer US, 2008.

[5] A. Artusi, F. Banterle, and D. Chetverikov, "A survey of specularity removal methods," Computer Graphics Forum, vol. 30, no. 8, pp. 2208-2230, 2011.

[6] S. A. Shafer, "Using color to separate reflection components," Color Research \& Application, vol. 10, no. 4, pp. 210-218, 1985.

[7] J. W. Park and K. H. Lee, "Inpainting highlights using color line projection," IEICE-Transactions on Information and Systems, vol. E90-D, pp. 250-257, Jan. 2007.

[8] Budianto and D. P. K. Lun, "Inpainting for fringe projection profilometry based on geometrically guided iterative regularization," IEEE Transactions on Image Processing, vol. 24, pp. 5531-5542, Dec 2015.

[9] F. Ortiz and F. Torres, "A new inpainting method for highlights elimination by colour morphology," in proceedings of 3rd International Conference on Advances in Pattern Recognition, pp. 368-376, Springer Berlin Heidelberg, 2005.

[10] P. Tan, S. Lin, L. Quan, and H.-Y. Shum, "Highlight removal by illumination-constrained inpainting," in proceedings of 9th IEEE International Conference on Computer Vision, vol. 1, pp. 164-169, Oct 2003. 
[11] G. Klinker, S. Shafer, and T. Kanade, "Using a color reflection model to separate highlights from object color," in proceedings of 1st International Conference on Computer Vision, pp. 145-150, 1991.

[12] G. J. Klinker, S. A. Shafer, and T. Kanade, "The measurement of highlights in color images," International Journal of Computer Vision, vol. 2, no. 1, pp. 7-32, 1988.

[13] G. J. Klinker, S. A. Shafer, and T. Kanade, "A physical approach to color image understanding," International Journal of Computer Vision, vol. 4, no. 1 , pp. $7-38,1990$.

[14] K. Schlüns and M. Teschner, "Analysis of 2D color spaces for highlight elimination in 3d shape reconstruction," in proceedings of the Asian Conference on Computer Vision II, pp. 801-805, 1995.

[15] K. Schlüns and M. Teschner, "Fast separation of reflection components and its application in 3D shape recovery," Color and Imaging Conference, no. 1, pp. 48-51, 1995.

[16] R. Bajcsy, S. W. Lee, and A. Leonardis, "Detection of diffuse and specular interface reflections and inter-reflections by color image segmentation," International Journal of Computer Vision, vol. 17, no. 3, pp. 241-272, 1996.

[17] S. P. Mallick, T. Zickler, P. N. Belhumeur, and D. J. Kriegman, "Specularity removal in images and videos: A PDE approach," in proceedings of 9th European Conference on Computer Vision, (Graz, Austria), pp. 550-563, Springer Berlin Heidelberg, 2006.

[18] J. Yang, Z. Cai, L. Wen, Z. Lei, G. Guo, and S. Z. Li, "A new projection space for separation of specular-diffuse reflection components in color images," in proceedings of 11th Asian Conference on Computer Vision, (Daejeon, Korea), pp. 418-429, Springer Berlin Heidelberg, 2013.

[19] J. Yang, L. Liu, and S. Z. Li, "Separating specular and diffuse reflection components in the HSI color space," in IEEE International Conference on Computer Vision Workshops, pp. 891-898, Dec 2013.

[20] Y. Akashi and T. Okatani, "Separation of reflection components by sparse non-negative matrix factorization," Computer Vision and Image Understanding, vol. 146, pp. 77-85, 2016.

[21] R. T. Tan and K. Ikeuchi, "Separating reflection components of textured surfaces using a single image," IEEE Transactions on Pattern Analysis and Machine Intelligence, vol. 27, pp. 178-193, Feb 2005.

[22] K. j. Yoon, Y. Choi, and I. S. Kweon, "Fast separation of reflection components using a specularity-invariant image representation," in International Conference on Image Processing, pp. 973-976, Oct 2006.

[23] H.-L. Shen and Q.-Y. Cai, "Simple and efficient method for specularity removal in an image," Applied Optics, vol. 48, pp. 2711-2719, May 2009.

[24] H.-L. Shen, H.-G. Zhang, S.-J. Shao, and J. H. Xin, "Chromaticity-based separation of reflection components in a single image," Pattern Recognition, vol. 41, no. 8, pp. 2461-2469, 2008.

[25] Y. Liu, Z. Yuan, N. Zheng, and Y. Wu, "Saturation-preserving specular reflection separation," in IEEE Conference on Computer Vision and Pattern Recognition (CVPR), pp. 3725-3733, June 2015. 
[26] Q. Yang, S. Wang, and N. Ahuja, "Real-time specular highlight removal using bilateral filtering," in 11th European Conference on Computer Vision, (Heraklion, Greece), pp. 87-100, Springer Berlin Heidelberg, Sept. 2010.

[27] Q. Yang, J. Tang, and N. Ahuja, "Efficient and robust specular highlight removal," IEEE Transactions on Pattern Analysis and Machine Intelligence, vol. 37, pp. 1304-1311, June 2015.

[28] H. Kim, H. Jin, S. Hadap, and I. Kweon, "Specular reflection separation using dark channel prior," in IEEE Conference on Computer Vision and Pattern Recognition, pp. 1460-1467, June 2013.

[29] J. Suo, D. An, X. Ji, H. Wang, and Q. Dai, "Fast and high quality highlight removal from a single image," IEEE Transactions on Image Processing, vol. 25, pp. 5441-5454, Nov 2016.

[30] H.-L. Shen and Z.-H. Zheng, "Real-time highlight removal using intensity ratio," Applied Optics, vol. 52, pp. 4483-4493, Jul 2013.

[31] S. W. Lee and R. Bajcsy, "Detection of specularity using colour and multiple views," Image and Vision Computing, vol. 10, no. 10, pp. 643-653, 1992.

[32] Y. Sato and K. Ikeuchi, "Temporal-color space analysis of reflection," Journal of Optical Society of America A, vol. 11, pp. 2990-3002, Nov 1994.

[33] S. Lin and H.-Y. Shum, "Separation of diffuse and specular reflection in color images," in proceedings of the IEEE Computer Society Conference on Computer Vision and Pattern Recognition., vol. 1, pp. 341-346, 2001.

[34] S. Lin, Y. Li, S. B. Kang, X. Tong, and H.-Y. Shum, "Diffuse-specular separation and depth recovery from image sequences," in proceedings of 7th European Conference on Computer Vision, (Copenhagen, Denmark), pp. 210-224, Springer Berlin Heidelberg, May 2002.

[35] Y. Weiss, "Deriving intrinsic images from image sequences," in Proceedings Eighth IEEE International Conference on Computer Vision. ICCV, vol. 2, pp. 68-75, 2001.

[36] R. Feris, R. Raskar, K.-H. Tan, and M. Turk, "Specular highlights detection and reduction with multi-flash photography," Journal of the Brazilian Computer Society, vol. 12, no. 1, pp. 35-42, 2006.

[37] A. Agrawal, R. Raskar, S. K. Nayar, and Y. Li, "Removing photography artifacts using gradient projection and flash-exposure sampling," ACM Transactions on Graphics, vol. 24, pp. 828-835, July 2005.

[38] T. Chen, M. Goesele, and H. P. Seidel, "Mesostructure from specularity," in IEEE Computer Society Conference on Computer Vision and Pattern Recognition (CVPR), vol. 2, pp. 1825-1832, 2006.

[39] Q. Yang, S. Wang, N. Ahuja, and R. Yang, "A uniform framework for estimating illumination chromaticity, correspondence, and specular reflection," IEEE Transactions on Image Processing, vol. 20, pp. 53-63, Jan 2011.

[40] C. Wang, S. I. Kamata, and L. Ma, "A fast multi-view based specular removal approach for pill extraction," in IEEE International Conference on Image Processing, pp. 4126-4130, Sept 2013.

[41] V. Prinet, M. Werman, and D. Lischinski, "Specular highlight enhancement from video sequences," in IEEE International Conference on Image Processing, pp. 558-562, Sept 2013. 
[42] H. Wang, C. Xu, X. Wang, Y. Zhang, and B. Peng, "Light field imaging based accurate image specular highlight removal," PLOS ONE, vol. 11, pp. 1-17, 062016.

[43] S. K. Nayar, X.-S. Fang, and T. Boult, "Separation of reflection components using color and polarization," International Journal of Computer Vision, vol. 21, no. 3, pp. 163-186, 1997.

[44] L. B. Wolff, "Classification of material surfaces using the polarization of specular highlights," in proceedings of SPIE - The International Society for Optical Engineering, vol. 1005, pp. 206-213, Jan. 1988.

[45] L. B. Wolff, "Polarization-based material classification from specular reflection," IEEE Transactions on Pattern Analysis and Machine Intelligence, vol. 12, pp. 1059-1071, Nov 1990.

[46] D. W. Kim, S. Lin, K.-S. Hong, and H. Shum, "Variational specular separation using color and polarization," in proceedings of the IAPR Workshop on Machine Vision Applications, 2002.

[47] G. A. Atkinson and E. R. Hancock, "Recovery of surface orientation from diffuse polarization," IEEE Transactions on Image Processing, vol. 15, pp. 1653-1664, June 2006.

[48] G. A. Atkinson and E. R. Hancock, "Two-dimensional BRDF estimation from polarisation," Computer Vision and Image Understanding, vol. 111, no. 2, pp. 126-141, 2008.

[49] V. Müller, "Polarization-based separation of diffuse and specular surfacereflection," in Verstehen akustischer und visueller Informationen, pp. 202 209, Springer Berlin Heidelberg, 1995.

[50] S. Umeyama and G. Godin, "Separation of diffuse and specular components of surface reflection by use of polarization and statistical analysis of images," IEEE Transactions on Pattern Analysis and Machine Intelligence, vol. 26, pp. 639-647, May 2004.

[51] B. Lamond, P. Peers, and P. Debevec, "Fast image-based separation of diffuse and specular reflections," in ACM SIGGRAPH Sketches, SIGGRAPH '07, (New York, NY, USA), ACM, 2007.

[52] L. Zhang, E. R. Hancock, and G. A. Atkinson, "Reflection component separation using statistical analysis and polarisation," in proceedings of 5th Iberian Conference, IbPRIA, (Las Palmas de Gran Canaria, Spain), pp. 476483, Springer Berlin Heidelberg, 2011.

[53] V. Bochko and J. Parkkinen, "Highlight analysis using a mixture model of probabilistic PCA," in proceedings of the 4 th WSEAS International Conference on Signal Processing, Robotics and Automation, ISPRA'05, (Stevens Point, Wisconsin, USA), pp. 15:1-15:5, World Scientific and Engineering Academy and Society (WSEAS), 2005.

[54] Z. Fu, R. T. Tan, and T. Caelli, "Specular free spectral imaging using orthogonal subspace projection," in 18th International Conference on Pattern Recognition (ICPR), vol. 1, pp. 812-815, 2006.

[55] P. Koirala, P. Pant, M. Hauta-Kasari, and J. Parkkinen, "Highlight detection and removal from spectral image," Journal of Optical Society of America A, vol. 28, pp. 2284-2291, Nov 2011. 